\author{
Michael Silva-Peñaherrera ${ }^{a, b}$ \\ (D) https://orcid.org/0000-0001-5133-181X \\ Pamela Merino-Salazar ${ }^{\mathrm{a}, \mathrm{b}}$ \\ (D) https://orcid.org/0000-0002-3796-4706 \\ Fernando G. Benavides ${ }^{\mathrm{b}, \boldsymbol{c}, \mathrm{d}}$ \\ (iD) https://orcid.org/0000-0003-0747-2660 \\ María López-Ruiz, \\ (DD https://orcid.org/0000-0003-3453-0408 \\ Antonio Ramón Gómez-García ${ }^{f}$ \\ (D) https://orcid.org/0000-0003-1015-1753 \\ a Universidad Internacional SEK. Quito, \\ Equador. \\ b Universitat Pompeu Fabra, Centro \\ de Pesquisas em Saúde Ocupacional. \\ Barcelona, Espanha. \\ ${ }^{\complement}$ Centro de Investigación Biomédica \\ en Red Epidemiología y Salud Pública \\ (CIBERESP). Madri, Espanha. \\ d Instituto de Pesquisa Hospital del Mar \\ Medical (IMIM). Barcelona, Espanha. \\ e Facultad Latinoamericana de Ciencias \\ Sociales. Salamanca, Espanha. \\ ${ }^{\mathrm{f}}$ Universidad Espiritu Santo. \\ Samborondon, Equador. \\ Contato: \\ Michael Silva-Peñaherrera \\ E-mail: \\ michael.silvap@gmail.com
}

Este estudo contou com apoio da Universidade Internacional SEK do Equador e da Universidad de Especialidades Espiritu Santo, da cidade de Guayaquil, Equador.

Os autores declaram não haver conflitos de interesses.

Os autores informam que este trabalho não foi baseado em dissertação ou tese e que foi apresentado no XXXVI Encontro Científico da Sociedade Espanhola de Epidemiologia e no XIII Congresso da Associação Portuguesa de Epidemiologia, em 2018, Lisboa, Portugal.

Recebido: 15/02/2019

Revisado: 03/05/2019

Aprovado: 23/07/2019

\section{Saúde do trabalhador no Equador: uma comparação com inquéritos sobre condições de trabalho na América Latina}

\author{
Occupational health in Ecuador: a comparison \\ with Latin-American surveys on working conditions
}

\section{Resumo}

Introdução: o Equador realizou recentemente seu primeiro inquérito sobre condições de trabalho. Objetivo: descrever as condições de trabalho e emprego e o estado de saúde dos trabalhadores equatorianos em uma amostra que permita comparação com inquéritos latino-americanos anteriores. Métodos: uma amostra de 1.713 trabalhadores foi selecionada do primeiro inquérito sobre condições de trabalho no Equador. Prevalências e intervalos de confiança de 95\% (IC95\%) foram calculados e comparados com inquéritos anteriores na Colômbia, Argentina, Chile, América Central e Uruguai. Resultados: homens foram frequentemente mais expostos a trabalho em condições perigosas, sendo as mais relatadas trabalho com ruído (81\% dos homens e $69 \%$ das mulheres) e com movimentos repetitivos (56\% dos homens e $48 \%$ das mulheres). Cerca de $31 \%$ dos homens e 19\% das mulheres trabalhavam mais de 40 horas por semana. Quase 11\% das mulheres e dos homens relataram uma autopercepção de saúde ruim. A prevalência de agravos ocupacionais foi a mais alta da região: 15\% para homens e 8,4\% para mulheres. Conclusão: este é um primeiro levantamento das condições de trabalho e emprego e do estado de saúde de trabalhadores no Equador. Harmonizar e aperfeiçoar os Inquéritos sobre Condições de Trabalho na América Latina deve ser uma meta prioritária para melhorar a vigilância em saúde do trabalhador na região.

Palavras-chave: saúde do trabalhador; sistema de informação em saúde; condições de trabalho; estado de saúde; Equador.

\begin{abstract}
Introduction: Ecuador has recently implemented its First Working Conditions Survey. Objective: to describe working and employment conditions and workers' health status in Ecuador in a sample that allows comparison with previous Latin American surveys. Methods: a sample of 1,713 workers was drawn from the First Working Conditions Survey in Ecuador. Prevalence and a 95\% confidence Interval (95\%CI) were calculated and compared with previous Latin American surveys in Colombia, Argentina, Chile, Central America, and Uruguay. Results: men were more often exposed to hazardous working conditions, with noise ( $81 \%$ of men and $69 \%$ of women), and repetitive movements (56\% and $48 \%$, respectively) being the most frequently reported. About $31 \%$ of men and $19 \%$ of women worked more than 40 hours per week. Almost $11 \%$ of both women and men reported poor self-perceived health status. The prevalence of occupational injury was the highest in the region: $15 \%$ for men and $8.4 \%$ for women. Conclusions: this is a first approach to the working and employment conditions and workers' health status in Ecuador. To harmonize and improve Working Conditions Surveys in Latin America should be a priority goal for enhancing regional occupational health surveillance.
\end{abstract}

Keywords: occupational health; health information system; working conditions; health status; Ecuador. 


\section{Introdução}

Emprego e condições de trabalho são fortes determinantes da saúde dos trabalhadores e de suas famílias ${ }^{1}$. Há crescentes evidências científicas mostrando que más condições de trabalho e emprego, como insegurança no trabalho e emprego precário, criam efeitos nocivos à saúde, absenteísmo e agravos ocupacionais, e produzem iniquidades em saúde na população ${ }^{2,3}$.

A concorrência global e a crescente necessidade de contenção de custos de produção têm pressionado para a "flexibilidade no trabalho", afetando a segurança e a saúde dos trabalhadores ${ }^{4,5}$. Além disso, como a globalização permite a terceirização, esse custo de saúde geralmente é transferido para países de renda baixa e média, onde a falta de políticas e de controle expõe os trabalhadores a más condições de trabalho e emprego. Com efeito, os países de renda alta têm cinco vezes menos acidentes de trabalho fatais e não fatais do que a média mundial e seis vezes menos do que a média da América Latina e do Caribe $^{6}$.

Em países de renda baixa e média, a escassez de informações confiáveis sobre condições de trabalho e saúde é uma das principais barreiras para o estabelecimento de políticas públicas adequadas $^{7,8}$. Vários programas internacionais de saúde e desenvolvimento reconheceram como meta prioritária a melhoria dos sistemas de informação em saúde ocupacional ${ }^{9,10}$.

Nesse sentido, os inquéritos sobre condições de trabalho (ICT) se consolidaram como uma ferramenta confiável para monitorar as condições de trabalho, emprego e saúde ${ }^{11}$. Na última década, vários países latino-americanos realizaram seu primeiro ICT ${ }^{12}$. O objetivo dessas pesquisas é subsidiar suas políticas nacionais. Esses esforços também permitiram as primeiras comparações entre países da região, oferecendo melhores evidências para elaboradores de políticas ${ }^{13}$.

O Equador realizou recentemente seu primeiro ICT (em espanhol I Encuesta sobre Condiciones de Seguridad y Salud en el Trabajo / I-ECSST), fornecendo um primeiro olhar sobre as condições de trabalho e de saúde pela perspectiva dos trabalhadores. O objetivo deste estudo é descrever as condições de trabalho e de emprego e o estado de saúde de trabalhadores em ocupações não agrícolas, cadastrados no sistema de seguridade social nas cidades de Quito e Guayaquil, em uma amostra que permite comparação com pesquisas latino-americanas anteriores (Colômbia, Argentina, Chile, América Central e Uruguai).

\section{Métodos}

Os dados utilizados foram obtidos da I-ECSST do Equador, realizada na capital equatoriana, Quito, e em Guayaquil, a cidade mais populosa do país, entre abril de 2016 e janeiro de 2017.

A I-ECSST foi uma pesquisa transversal baseada em uma amostra de trabalhadores assalariados com 18 ou mais anos de idade, cadastrados no sistema de previdência social, de todos os setores de atividade econômica e residentes em Quito ou Guayaquil. Foi selecionada uma amostra representativa de 1.790 trabalhadores (741 de Quito e 1.049 de Guayaquil) por meio de um procedimento de amostragem aleatória em múltiplos estágios, estratificado por bairro e com base no censo populacional e domiciliar do Equador de 2010. O questionário presencial foi aplicado na casa dos trabalhadores, com apenas um trabalhador sendo entrevistado por domicílio. Os detalhes da pesquisa estão publicados no relatório oficial ${ }^{14}$.

Para comparar os resultados da I-ECSST com ICT anteriores da Colômbia (2007) ${ }^{15}$, Argentina (2009) ${ }^{16}$, Chile (2009-2010) ${ }^{17}$, América Central $(2011)^{18}$ e Uruguai $(2012)^{19}$, conforme realizado em estudo anterior ${ }^{13}$, selecionamos uma amostra de trabalhadores cadastrados no sistema de seguridade social, com idade entre 18 e 64 anos e em ocupações não agrícolas. A amostra final foi composta por 1.713 trabalhadores de Quito e Guayaquil (848 mulheres e 865 homens).

As variáveis que permitiram a comparação entre a I-ECSST e outros ICT latino-americanos foram: gênero, idade (nas faixas de 18-34 anos, 35-49 anos e 50-64 anos), escolaridade (categorizada em fundamental, secundária e universitária), setor econômico (categorizado em indústria, construção e serviços) e categorias ocupacionais (categorizadas em gerentes, profissionais, técnicos, pessoal administrativo, pessoal de serviços e vendas, operários qualificados e operários não qualificados).

Com relação às condições de trabalho, selecionamos as seguintes variáveis de estudo: ruído, vibrações, manuseio e inalação de substâncias químicas perigosas, agentes biológicos, movimentação movimentação manual de carga e movimentos repetitivos. Seguindo os mesmos critérios do estudo anterior, as respostas foram dicotomizadas em "exposto" e "não exposto", nas quais qualquer resposta diferente de "nunca" significa "exposto". Para condições de emprego foi possível selecionar apenas a variável carga horária semanal $(<30$ horas, 30-40 horas, $>40$ horas).

Em relação a condições de saúde, duas variáveis foram selecionadas. A autopercepção do estado de saúde foi obtida solicitando aos entrevistados que 
descrevessem sua saúde geral como "excelente", "muito boa", "boa", "regular", "ruim" ou "péssima". Os resultados foram agregados em duas categorias, onde as respostas "regular", "ruim" ou "péssima" indicavam autopercepção de saúde ruim. Os agravos ocupacionais foram avaliadas perguntando se os entrevistados haviam sofrido ou não um acidente de trabalho nos 12 meses anteriores.

Para cada país, foram calculadas a prevalência e um intervalo de confiança de 95\% (IC95\%) da exposição a más condições de emprego e de trabalho e para problemas relacionados à saúde . Todas as análises foram estratificadas por gênero e realizadas com o software estatístico SPSS23.

\section{Resultados}

A maioria dos trabalhadores não agrícolas com cobertura de seguridade social e residentes em Quito ou Guayaquil (Tabela 1) era jovem, com $11 \%$ das mulheres e $13 \%$ dos homens acima de 50 anos de idade, e a grande maioria possuía instrução secundária e universitária. Tanto homens quanto mulheres trabalhavam em sua maioria no setor de serviços
(89\% e 82\%, respectivamente) em ocupações relacionadas a prestação de serviços e de vendas e ao apoio administrativo (67\% e $57 \%$, respectivamente).

Em relação a condições de emprego, no Equador, 19,3\% (IC95\%: 16,7\% - 22,0\%) das mulheres e 31,4\% (IC95\%: 28,3\% - 34,6\%) dos homens trabalhavam mais de 40 horas por semana, os menores percentuais da região (por exemplo, comparado com o Chile: $63,5 \%$ das mulheres e $83,6 \%$ dos homens). Considerando as condições de trabalho, a exposição a ruído foi o risco mais prevalente entre os trabalhadores equatorianos: 81,2\% (IC95\%: 78,5\% $83,8 \%$ ) dos homens e 69,0\% (IC95\%: 65,9\% -72,1\%) das mulheres, seguido de movimentos repetitivos: 56,1\% (IC95\%: 52,8\% -59,4\%) e 48,1\% (IC95\%: $44,8 \%$ - 51,5\%), respectivamente. Em todos os países, a frequência de exposição a risco foi maior entre os homens (Tabela 2). Além disso, tal exposição foi maior comparando os resultados de Equador com a de outros países da região; por exemplo, na América Central, a exposição a ruído foi de $54,1 \%$ para mulheres e $62,0 \%$ para homens; a substâncias químicas no ar foi de $30,8 \%$ e $39,4 \%$, respectivamente, e a agentes biológicos foi de $9,9 \%$ e 9,4\%, respectivamente.

Tabela 1 Características sociodemográficas de trabalhadores assalariados não agrícolas, segundo o gênero, em Quito e Guayaquil, Equador, 2016-2017 (n=1.713)

\begin{tabular}{|c|c|c|c|c|}
\hline \multirow{2}{*}{ Variável } & \multicolumn{2}{|c|}{ Mulheres $n=848$} & \multicolumn{2}{|c|}{ Homens $n=865$} \\
\hline & $\%$ & (IC95\%) & $\%$ & (IC95\%) \\
\hline \multicolumn{5}{|l|}{ Idade } \\
\hline 18-34 anos & 52,5 & $(49,1-55,8)$ & 45,1 & $(41,8-48,4)$ \\
\hline 35-49 anos & 36,9 & $(33,7-40,2)$ & 42,2 & $(38,9-45,5)$ \\
\hline 50-64 anos & 10,6 & $(8,5-12,7)$ & 12,7 & $(10,5-14,9)$ \\
\hline \multicolumn{5}{|l|}{ Escolaridade* $^{*}$} \\
\hline Fundamental & 5,8 & $(4,2-7,3)$ & 6,7 & $(5,0-8,4)$ \\
\hline Secundária & 36,8 & $(33,5-40,2)$ & 46,0 & $(42,7-49,3)$ \\
\hline Universitária & 56,8 & $(53,5-60,2)$ & 45,9 & $(42,6-49,2)$ \\
\hline \multicolumn{5}{|l|}{ Atividade econômica } \\
\hline Indústria & 7,9 & $(6,1-9,7)$ & 9,0 & $(7,1-10,9)$ \\
\hline Construção & 3,1 & $(1,9-4,2)$ & 9,4 & $(7,4-11,3)$ \\
\hline Serviços & 89,0 & $(86,9-91,1)$ & 81,6 & $(79,0-84,2)$ \\
\hline \multicolumn{5}{|l|}{ Categorias ocupacionais } \\
\hline Gerentes & 4,4 & $(3,0-5,7)$ & 6,0 & $(4,4-7,6)$ \\
\hline Profissionais & 7,3 & $(5,6-9,1)$ & 4,6 & $(3,2-6)$ \\
\hline Técnicos & 7,7 & $(5,9-9,5)$ & 11,2 & $(9,1-13,3)$ \\
\hline Pessoal administrativo & 25,1 & $(22,2-28,0)$ & 12,6 & $(10,4-14,8)$ \\
\hline Trabalhadores de serviços e de vendas & 42.3 & $(39-45.7)$ & 42.3 & $(39,0-45.6)$ \\
\hline Trabalhador manual qualificado & 4.9 & $(3.5-6.4)$ & 13.6 & $(11.4-15.9)$ \\
\hline Trabalhador manual não qualificado & 8.3 & $(6.4-10.1)$ & 9.6 & $(7.6-11.6)$ \\
\hline
\end{tabular}

*dados não disponíveis: mulheres $\mathrm{n}=5$; homens $\mathrm{n}=12$ 
Tabela 2 Condições de emprego e trabalho e estado de saúde, segundo o gênero, de trabalhadores não agrícolas, com idade entre 18 e 64 anos, no Equador, Colômbia, Argentina, Chile, América Central e Uruguai

\begin{tabular}{|c|c|c|c|c|c|c|c|c|c|c|c|c|}
\hline \multirow[b]{2}{*}{ Variável } & \multicolumn{2}{|r|}{ Equador } & \multicolumn{2}{|c|}{ Colômbia } & \multicolumn{2}{|c|}{ Argentina } & \multicolumn{2}{|r|}{ Chile } & \multicolumn{2}{|c|}{ América Central } & \multicolumn{2}{|r|}{ Uruguai } \\
\hline & $\%$ & (IC95\%) & $\%$ & (IC95\%) & $\%$ & (IC95\%) & $\%$ & (IC95\%) & $\%$ & (IC95\%) & $\%$ & (IC95\%) \\
\hline Mulheres & & $n=848$ & & $n=366$ & & $n=2646$ & & $y=1540$ & & $n=1034$ & & $n=358$ \\
\hline \multicolumn{13}{|l|}{ Condições de emprego } \\
\hline \multicolumn{13}{|l|}{ Carga horária semanal } \\
\hline$<30 \mathrm{~h}$ & 5,4 & $(3,9-6,9)$ & - & & 21,8 & $(16,6-27,1)$ & 5,2 & $(3,2-7,2)$ & 12,4 & $(9,9-14,9)$ & 17,5 & $(12,6-22,5)$ \\
\hline $30-40 h$ & 75,2 & $(72,3-78,1)$ & - & & 29,0 & $(24,3-33,6)$ & 31,3 & $(26,8-35,8)$ & 27,1 & $(24,0-30,3)$ & 41,4 & $(35,1-47,6)$ \\
\hline$>40 h$ & 19,3 & $(16,7-22,0)$ & - & & 49,2 & $(44,1-54,4)$ & 63,5 & $(58,8-68,2)$ & 60,5 & $(57-63,9)$ & 41,1 & $(34,8-47,4)$ \\
\hline \multicolumn{13}{|l|}{ Condições de trabalho } \\
\hline Ruído* & 69,0 & $(65,9-72,1)$ & 23,3 & $(19,0-27,6)$ & 14,9 & $(11,8-18)$ & 22,5 & $(18,8-26,3)$ & 54,1 & $(50,7-57,6)$ & 22,3 & $(17,3-27,4)$ \\
\hline Vibrações\$ & 12,0 & $(9,8-14,2)$ & 6,8 & $(4,3-9,4)$ & 5,5 & $(3,6-7,4)$ & 11,2 & $(8,2-14,3)$ & 25,1 & $(22,2-28,1)$ & 7,9 & $(4,9-10,9)$ \\
\hline $\begin{array}{l}\text { Manuseio de } \\
\text { substâncias químicas } \\
\text { nocivas }^{5}\end{array}$ & 19,2 & $(16,6-21,9)$ & 8,8 & $(5,9-11,7)$ & 11,9 & $(6,7-17,1)$ & 9,6 & $(7,1-12,2)$ & 16,9 & $(14,4-19,5)$ & 6,4 & $(3,7-9,1)$ \\
\hline $\begin{array}{l}\text { Inalação de substâncias } \\
\text { químicas nocivas" }\end{array}$ & 38,8 & $(35,5-42,1)$ & 19,2 & $(15,2-23,3)$ & 2,6 & $(1,6-3,6)$ & 7,3 & $(4,8-9,7)$ & 30,8 & $(27,7-34,0)$ & 14,0 & $(9,7-18,2)$ \\
\hline Agentes biológicos* & 30,2 & $(27,1-33,3)$ & 8,2 & $(5,4-11)$ & 9,4 & $(6,5-12,3)$ & 17,7 & $(14,0-21,4)$ & 9,9 & $(7,8-11,9)$ & 12,3 & $(8,3-16,2)$ \\
\hline $\begin{array}{l}\text { Movimentação manual } \\
\text { de carga* }\end{array}$ & 36,7 & $(33,4-39,9)$ & 13,4 & $(9,9-16,9)$ & 24,1 & $(19,9-28,3)$ & 24,0 & $(20,1-27,9)$ & 13,9 & $(11,5-16,2)$ & 27,8 & $(22,3-33,4)$ \\
\hline $\begin{array}{l}\text { Movimentos } \\
\text { repetitivos }^{\varsigma}\end{array}$ & 48,1 & $(44,8-51,5)$ & 84,4 & $(80,7-88,1)$ & 50,8 & $(45,6-56)$ & 54,7 & $(49,9-59,5)$ & 73,7 & $(70,7-76,7)$ & 51,3 & $(45,0-57,8)$ \\
\hline \multicolumn{13}{|l|}{ Estado de saúde } \\
\hline $\begin{array}{l}\text { Autopercepção de } \\
\text { saúde ruim }{ }^{+}\end{array}$ & 10,8 & $(8,8-12,9)$ & 5,5 & $(3,1-7,8)$ & - & & 33,4 & $(28,4-38,3)$ & 24,3 & $(21,3-27,2)$ & - & \\
\hline Lesões ocupacionais ${ }^{++}$ & 8,4 & $(6,5-10,2)$ & 3,8 & $(1,9-5,8)$ & - & & 7,4 & $(4,6-10,2)$ & 4,5 & $(3,1-6,0)$ & - & \\
\hline Homens & & $n=865$ & & $n=455$ & & $n=4402$ & & $n=2310$ & & $n=1632$ & & $n=498$ \\
\hline \multicolumn{13}{|l|}{ Condições de emprego } \\
\hline \multicolumn{13}{|l|}{ Carga horária semanal } \\
\hline$<30 h$ & 2,5 & $(1,5-3,6)$ & - & & 5,4 & $(3,4-7,5)$ & 0,9 & $(0,2-1,5)$ & 5,6 & $(4,0-7,2)$ & 7,9 & $(4,6-11,1)$ \\
\hline $30-40 h$ & 66,0 & $(62,8-69,2)$ & - & & 18,3 & $(15,6-21)$ & 15,5 & $(12,8-18,2)$ & 21,0 & $(18,6-23,5)$ & 34,1 & $(28,4-39,8)$ \\
\hline$>40 \mathrm{~h}$ & 31,4 & $(28,3-34,6)$ & - & & 76,3 & $(73,1-79,4)$ & 83,6 & $(80,9-86,4)$ & 73,3 & $(70,6-76,0)$ & 58,1 & $(52,1-64,0)$ \\
\hline \multicolumn{13}{|l|}{ Condições de trabalho } \\
\hline Ruído* & 81,2 & $(78,5-83,8)$ & 45,2 & $(40,6-49,7)$ & 22,5 & $(19,7-25,2)$ & 45,6 & $(41,1-50,1)$ & 62,0 & $(59,2-64,7)$ & 30,7 & $(25,6-35,8)$ \\
\hline Vibrações\$ & 35,8 & $(32,6-39,0)$ & 30,8 & $(26,6-35,1)$ & 17,8 & $(15,4-20,2)$ & 42,1 & $(37,6-46,5)$ & 37,6 & $(34,79-40,4)$ & 30,0 & $(24,8-35,2)$ \\
\hline $\begin{array}{l}\text { Manuseio de } \\
\text { substâncias químicas } \\
\text { nocivas }^{5}\end{array}$ & 22,2 & $(19,4-25,0)$ & 22,1 & $(18,3-25,9)$ & 12,5 & $(10,7-14,4)$ & 19,1 & $(15,9-22,4)$ & 18,4 & $(16,2-20,6)$ & 18,3 & $(13,9-22,8)$ \\
\hline $\begin{array}{l}\text { Inalação de substâncias } \\
\text { químicas nocivas* }\end{array}$ & 57,5 & $(54,2-60,8)$ & 50,9 & $(46,3-55,5)$ & 11,9 & $(9,9-13,8)$ & 32,9 & $(28,9-36,9)$ & 39,4 & $(36,6-42,2)$ & 31,4 & $(26,1-36,8)$ \\
\hline Agentes biológicos* & 31,7 & $(28,6-34,8)$ & 8,2 & $(5,6-10,7)$ & 6,2 & $(4,7-7,6)$ & 9,2 & $(7,1-11,3)$ & 9,4 & $(7,7-11,2)$ & 15,1 & $(10,7-19,5)$ \\
\hline $\begin{array}{l}\text { Movimentação manual } \\
\text { de carga* }\end{array}$ & 58,6 & $(55,3-61,9)$ & 34,4 & $(30,0-38,7)$ & 44,9 & $(41,2-48,5)$ & 39,9 & $(35,7-44,1)$ & 36,6 & $(33,9-39,3)$ & 45,5 & $(39,5-51,5)$ \\
\hline $\begin{array}{l}\text { Movimentos } \\
\text { repetitivos }^{s}\end{array}$ & 56,1 & $(52,8-59,4)$ & 76,9 & $(73,0-80,8)$ & 62,8 & $(59,1-66,5)$ & 60,0 & $(55,4-64,7)$ & 77,3 & $(74,9-79,9)$ & 58,6 & $(52,6-64,6)$ \\
\hline \multicolumn{13}{|l|}{ Estado de saúde } \\
\hline $\begin{array}{l}\text { Autopercepção de } \\
\text { saúde ruim }{ }^{+}\end{array}$ & 10,9 & $(8,8-12,9)$ & 4,2 & $(2,3-6,0)$ & - & & 16,6 & $(13,4-19,7)$ & 19,1 & $(16,9-21,4)$ & - & \\
\hline Lesões ocupacionais ${ }^{++}$ & 15,0 & $(12,6-17,4)$ & 9,9 & $(7,1-12,6)$ & - & & 6,5 & $(4,7-8,3)$ & 4,9 & $(3,7-6,1)$ & - & \\
\hline
\end{tabular}

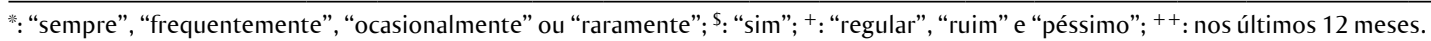


Quanto a problemas de saúde, os homens equatorianos tiveram mais propensão a sofrer lesões ocupacionais do que as mulheres: 15\% (IC95\%: 12,6\%-17,4\%) contra. 8,4\% (IC95\%: 6,5\%-10,2\%), e, conforme relatado em outros países, apresentaram a maior prevalência na região, seguido por Colômbia (9,9\% dos homens e 3,8\% das mulheres), Chile $(6,5 \%$ dos homens e 7,4\% das mulheres) e América Central (4,9\% dos homens e 4,5\% das mulheres). Por fim, quase $11 \%$ de mulheres e homens no Equador relataram autopercepção de saúde ruim, uma prevalência abaixo do Chile e da América Central.

\section{Discussão}

Este estudo lança um primeiro olhar sobre as condições de trabalho e a saúde de trabalhadores formais em duas das cidades mais populosas do Equador, fornecendo uma primeira perspectiva comparativa em relação a resultados anteriores de ICT em países da América Latina.

Nossa amostra apresentou uma distribuição semelhante à da região, com uma pequena diferença devida à maior proporção de trabalhadores no setor de serviços: 81,6\% dos homens no Equador contra $78,1 \%$ dos homens na América Central ${ }^{13}$. Uma possível razão para isso seria o fato de o ICT equatoriano ter sido implementado nas duas maiores cidades comerciais e administrativas do país.

O Equador tem a menor porcentagem de homens e mulheres trabalhando mais de 40 horas por semana na região. A média da carga horária semanal da população formalmente empregada no Equador foi 43,1 para homens e 41,3 para mulheres, semelhante à média de 42 horas encontrada para a América Latina, relatada em 2013, com base em pesquisas domiciliares nacionais ${ }^{20}$, embora a população formalmente empregada tenha relatado uma média mais baixa em outros ICT na região. Esse resultado pode estar relacionado às leis de muitos países da região que permitem cargas horárias semanais além do máximo recomendado pela Organização Internacional do Trabalho ${ }^{21}$.

Em relação a condições de trabalho, constatamos alguns casos de exposição maior em comparação a outros países da região. Essa alta prevalência no Equador pode ter sido superestimada dado o critério usado para agregar as respostas, pelo qual uma escala Likert de cinco pontos foi dicotomizada e qualquer resposta diferente de "nunca" significava "exposto". No entanto, também foi superior à Colômbia, onde foi utilizada uma escala Likert de cinco pontos semelhante. São necessárias mais pesquisas para explicar essas diferenças.
Ao contrário do Chile e da América Central, não encontramos diferenças segundo o gênero em relação à autopercepção de saúde ruim. Esse resultado pode estar relacionado à sensibilidade dessa medida ao ambiente cultural. Por exemplo, em um estudo longitudinal europeu não foram encontradas diferenças substanciais em autopercepção de saúde segundo o gênero ${ }^{22}$. Seja como for, o índice equatoriano ficou acima da Colômbia e abaixo do Chile e da América Central.

O Equador apresentou a maior prevalência de agravos ocupacionais na região. Esses resultados foram muito superiores aos relatados pelo sistema de seguridade social do Equador $^{23}$, que mal chegam a $0,62 \%$. Tal discrepância nos alertou para a possibilidade de uma significativa subnotificação nos números oficiais do país. É um problema comum a muitos países ${ }^{6}$.

Há algumas limitações deste estudo que devem ser consideradas. Primeiro, a amostra incluiu apenas trabalhadores cadastrados no sistema de seguridade social, em ocupações não agrícolas e residentes em Quito ou Guayaquil. Isso pode ter causado um viés de seleção, pois embora essas duas cidades tenham o maior contingente de trabalhadores com seguridade social $(62 \%)^{23}$, o emprego informal não foi considerado. De acordo com o relatório sobre o mercado de trabalho equatoriano de 2017, o emprego informal responde por $46 \%$ da população economicamente ativa ${ }^{24}$. É um problema comum no mercado de trabalho da região, variando de $83,1 \%$ na Bolívia a 40,5\% no Chile ${ }^{25}$. Na verdade, a comparação anterior entre os ICT da América Latina também excluiu o emprego informal. A segunda limitação reside nas diferenças entre os questionários (referentes aos itens incluídos e às categorias de resposta) utilizadas para os ICT latino-americanos que limitaram o número de variáveis que pudemos comparar. Por exemplo, excluímos os resultados psicossociais em nosso estudo porque as categorias de perguntas e respostas tinham comparabilidade limitada.

Apesar dessas limitações, essas são as melhores informações disponíveis no Equador até o momento, e são um primeiro passo para diagnosticar as condições de saúde e trabalho no país. Este estudo também apresenta uma comparação preliminar entre a saúde ocupacional no Equador e em outros países da América Latina, fornece informações básicas para a vigilância de saúde e de condições de trabalho no país e aprimora as informações de vigilância epidemiológica da região. Além disso, reforça a necessidade de se harmonizar e melhorar os ICT na América Latina $^{26}$, bem como a vigilância de saúde ocupacional global ${ }^{27}$. Neste estudo também se reconhece a necessidade de se estabelecer políticas públicas adequadas de saúde do trabalhador. 


\section{Agradecimentos}

Os autores agradecem a Amy Murphy pela revisão do texto original em inglês.

\section{Contribuição dos autores}

Silva-Peñaherrera M e G Benavides F contribuíram com a concepção do estudo, a análise e a interpretação dos dados e a redação do manuscrito. Merino-Salazar P, López-Ruiz M e Gómez-García AR fizeram contribuições substanciais para a concepção do estudo, a interpretação dos resultados e a revisão do manuscrito. Todos os autores revisaram criticamente a versão final do artigo e assumem integral responsabilidade por seu conteúdo.

\section{Referências}

1. Benach J, Muntaner C, Solar O, Santana V, Quinlan M. Introduction to the who Commission on Social Determinants of Health Employment Conditions Network (Emconet) Study, with a Glossary on Employment Relations. Int J Heal Serv. 2010;40(2):195-207. doi:10.2190/HS.40.2.a

2. World Health Organization. Social determinants of health: the solid facts [Internet]. Geneva; 2003 [citado em 11 jun 2020]. Disponível em: http://www.euro.who.int/_data/assets/pdf_ file/0005/98438/e81384.pdf

3. Benavides FG, Benach J, Muntaner C, Delclos GL, Catot N, Amable M. Associations between temporary employment and occupational injury: what are the mechanisms? Occup Environ Med. 2006;63(6):416-21. doi:10.1136/oem.2005.022301

4. Kawachi I. Globalization and Workers' Health. Ind Health. 2008;46(5):421-3. doi:10.2486/ indhealth.46.421

5. Lucchini RG, London L. Global occupational health: current challenges and the need for urgent action. Ann Glob Health. 2014;80(4):251-6. doi:10.1016/j.aogh.2014.09.006

6. Hämäläinen P, Takala J, Kiat TB. Global Estimates of Occupational Accidents and Work-Related Illnesses 2017. Singapore: Workplace Safety and Health Institute; 2017.

7. Wegman DH, Hogstedt C. If it's not counted it didn’t happen! Occup Environ Med. 2014;71(7):457-8. doi:10.1136/oemed-2014-102223

8. González Alvarez ME, Guzmán-Quilo MC. Advances in Occupational Health in Guatemala. Ann Glob Health. 2018;84(3):334-7. doi:10.29024/ aogh.2319

9. International Labour Office. Global Strategy on Occupational Safety and Health [Internet]. Geneva; 2004 [citado em 16 dez 2017]. Disponível em: http://www.ilo.org/wcmsp5/groups/public/---ed_ protect/---protrav/---safework/documents/policy/ wcms_107535.pdf

10. Organización Iberoamericana de Seguridad Social. II Estrategia Iberoamericana de Seguridad y Salud en el Trabajo 2015-2020. Madrid; 2015.
11. European Foundation for the Improvement of Living and Working Conditions. Sixth European Working Conditions Survey - Overview

Report (2017 Update). Loughlinstown; 2017. doi:10.2806/422172

12. Merino-Salazar P, Artazcoz L, Campos-Serna J, Gimeno D, Benavides FG. National working conditions surveys in Latin America: comparison of methodological characteristics. Int J Occup Environ Health. 2015;21(3):266-74. doi:10.1179/20 49396715Y.0000000004

13. Merino-Salazar P, Artazcoz L, Cornelio C, Itatí Iñiguez MJ, Rojas M, Martínez-Iñigo D, et al. Work and health in Latin America: results from the working conditions surveys of Colombia, Argentina, Chile, Central America and Uruguay. Occup Environ Med. 2017;74(6):432-9. doi:10.1136/oemed-2016-103899

14. Gómez-García AR, Merino-Salazar PA, SilvaPeñaherrera GM, Suasnavas Bermúdez PR, Vilaret Serpa A. I Encuesta Sobre Condiciones de Seguridad y Salud En El Trabajo: Quito. Quito: Universidad Internacional SEK; 2017.

15. Colombia. Ministerio de la Protección Social. Primera Encuesta Nacional de Condiciones de Salud y Trabajo En El Suistema General de Riesgos Profesionales. Bogotá; 2007.

16. Organización Iberoamericana de Seguridad Social. 1a Encuesta Nacional a Trabajadores Sobre Empleo, Trabajo, Condiciones y Medio Ambiente Laboral [Internet]. Madrid; 2012 [citado em 19 abr 2019]. Disponível em: http://publicaciones.srt.gob. ar/Publicaciones//2013/EncuestaNac2009.pdf

17. Chile. Ministerio de Salud. Primera Encuesta Nacional de Empleo, Trabajo, Salud, y Calidad de Vida de los Trabajadores y Trabajadoras en Chile (ENETS 2009-2010) [citado em 18 abr 2019]. Disponível em: https://www.dt.gob.cl/portal/1629/ articles-99630_recurso_1.pdf

18. Benavides FG, Wesseling C, Delclos GL, Felknor S, Pinilla J, Rodrigo F. Working conditions and health in Central America: a survey of 12024 workers in six countries. Occup Environ Med. 2014;71(7):459-65. doi:10.1136/oemed-2013-101908 
19. Martinez Iñigo D. Encuesta Sobre Condiciones de Trabajo, Seguridad y Salud Laboral En Uruguay. Madrid: OISS; 2013.

20. Comisión Económica para América Latina y el Caribe. Panorama Social de América Latina [Internet]. Santiago de Chile; 2013 [citado em $29 \mathrm{dez}$ 2017]. Disponível em: https://repositorio.cepal.org/ bitstream/handle/11362/35904/1/S2013868_es.pdf

21. International Labour Office. Working Conditions Laws Database [Internet]. Geneva; 2012 [citado em 11 jun 2020]. Disponível em: http://www.ilo.org/ dyn/travail

22. Jylhä M, Guralnik JM, Ferrucci L, Jokela J, Heikkinen E. Is Self-Rated Health Comparable Across Cultures and Genders? J Gerontol Soc Sci [Internet]. 1998 [citado em 18 dez 2017];53(3):44-52. Disponível em: https:/academic.oup.com/psychsocgerontology/ article/53B/3/S144/545419

23. Instituto Ecuatoriano de Seguridad Social. Boletín Estadístico Número 20 [Internet]. Quito; 2014 [citado em 11 jun 2020]. Disponível em: https:// www.iess.gob.ec/documents/10162/8421754/ BOLETIN+ESTADISTICO+20+2014.pdf
24. Instituto Nacional de Estadística y Censos (Ecuador). Reporte de Economía Laboral [Internet]. 2017 [citado em 11 jun 2020]. Disponível em: http://www.ecuadorencifras.gob.ec/documentos/ web-inec/EMPLEO/2017/Septiembre/Informe_ Economia_laboral-sep17.pdf

25. International Labour Office. Women and men in the informal economy: a statistical picture. 3a ed. Geneva; 2018.

26. Benavides FG, Merino-Salazar P, Cornelio C, Assunção AA, Agudelo-Suárez AA, Amable M, et al. Cuestionario básico y criterios metodológicos para las Encuestas sobre Condiciones de Trabajo, Empleo y Salud en América Latina y el Caribe. Cad Saude Publica. 2016;32(9):1-13. doi:10.1590/0102311x00210715

27. Merino-Salazar P, Cornelio C, Lopez-Ruiz M, Benavides FG. Propuesta de indicadores para la vigilancia de la salud ocupacional en América Latina y el Caribe. Rev Panam Salud Publica. 2018; 42:1-9. doi:10.26633/RPSP.2018.125 